\title{
Design, modeling and simulation of a three-layers piezoelectric cantilevered actuator with collocated sensor
}

\author{
Patrick Rougeot*, Abdenbi Mohand-Ousaid, Dominique Gendreau, \\ Mounir Hammouche and Micky RaKotondrabe \\ Automatic Control and MicroMechatronic Systems (AS2M) dept of FEMTO-ST Institute \\ UBFC, Université de Franche-Comté, ENSMM, CNRS, UTBM, \\ 24, rue Alain Savary Besançon, France; \\ *: corresponding author: mrakoton@femto-st.fr
}

\begin{abstract}
A new piezoelectric actuator with collocated sensor is designed, modeled and simulated. The structure has three piezoelectric layers where the two external layers serve for the actuation by a convenient application of electrical potentials, and the middle layer serves as the sensor. After presenting the principle of the structure, a model is developed for the actuator and as well as for the sensor. Then simulation is carried out to evaluate their performances. The novel structure is very promising for applications that require control and automation, especially in situations where the use of sensors is unfeasible or difficult.
\end{abstract}

Keywords: Piezoelectric actuator, piezoelectric sensor, collocated actuation and sensing, PVDF, PZT, modeling.

\section{INTRODUCTION}

Piezoelectric actuators (or Piezoactuators) are known for their ability to provide precise positioning ${ }^{1-4}$. They permit a very high positioning resolution and a high bandwidth. Furthermore, as they are powered electrically, they can be integrated easily. Finally, piezoelectric materials can also be used for measurement making possible the integration of actuation and sensing in the same system. One of the most used structures for piezoactuators are cantilevers. When subjected to a voltage, it bends. Two piezoelectric cantilevers are often used to constitute a piezoelectric microgripper that can pick, transport and place small objects during micromanipulation or microassembly tasks ${ }^{5,6}$. A major advantage of piezoelectric microgripper is the possibility to control the manipulation force with one cantilever and the displacement with the other cantilever ${ }^{7-9}$. Controlling the force or the displacement (bending of the actuator) requires however a real-time measurement of the signal.

Measuring the signal (force or displacement) in piezoactuator based precise positioning systems is however very challenging. Because of the compact sizes of the systems and the performances required, it is difficult or even impossible to find the appropriate sensors. In fact, sensors having the required precision and bandwidth for such microactuators are bulky and very expensive (optical sensors, ...) ${ }^{10}$ whilst embeddable sensors (strain gage, capacitive, ...) does not necessarily have the performances (range, precision, ...) ${ }^{11-13}$. This reason has particularly led to the raise of feedforward control architectures in piezoactuators these last fifteen years, i.e. controlling the actuators without using sensors ${ }^{2-4,14-24}$. The main limitation of feedforward control is however the lack of robustness relative to internal disturbances (model uncertainties) and to external disturbances. An endeavor consists therefore in trying to develop new approaches of measurements with ease of integration feature and which are capable of tracking the performances of the actuator. We focus in this paper on the measurement of signals in piezoelectric cantilever actuators.

In ${ }^{25}$, a piezoelectric sensor has been used in a piezoactuator. In fact, the electrodes on the surface of the cantilever has been split into two parts: one part is for the actuation and the other part is for the sensing. Fully integrated, the measurement principle has the same performances in term of bandwidth than the actuation since they use the same transduction. However, as the surface of the electrodes for the actuation has been reduced in order to create electrodes for sensing, the performance of the actuator in term of range is also reduced. Also, 
the surface of the electrodes for sensing is too small and provides very small amount of electrical charge making tricky its exploitation.

Another way to fully integrate a measurement system in a cantilever piezoactuator is to employ the same electrodes for actuation and for sensing simultaneously. Called piezoelectric self-sensing, this technique suggests more performances in term of actuation (more range of displacement) relative to the previous technique. Furthermore, if the self-sensing is appropriately designed, the measurement could be more sensitive. Indeed it offers more electrical charge than the previous technique for the sensing because of the larger electrodes surface. However, due to the internal leakage of the piezoelectric material, self-sensing is efficient at high frequency making it initially used for vibrations damping ${ }^{26-29}$. Later on, new self-sensing techniques for low frequency and constant displacement ${ }^{30}$ and for force ${ }^{31}$ signals for one degree of freedom (1-DOF) piezoactuator have been proposed. The techniques have later been extended to measure the displacement at low and high frequency ${ }^{32}$, and then the displacement and the force simultaneously ${ }^{33}$, making possible the robust feedback control of the 1-DOF actuator. Finally in ${ }^{34}$, a new electrical circuit for self-sensing for 2-DOF piezoactuators has been proposed. Piezoelectric self-sensing permitting to measure the signals at low and at high frequency is not well settled and is still under research because of the internal leakages, the limited performances of the electrical circuit components (bias current, dielectric absorption...) ${ }^{35,36}$ and the couplings between the actuation and sensing electrodes. The endeavor is focused on the improvement of the electrical circuit as well as on the modeling and on the observer which should account for these leakages, limitation and couplings.

Another interesting way to measure the cantilever piezoactuator is to introduce a piezoelectric layer for sensing inside the same cantilever. In this case, the layer for the sensor should have negligible stiffness relative to the stiffness of the actuator. Polyvinylidene fluoride (PVDF) piezoelectric polymer is a good candidate for such sensing layer as it has a very high compliance. It has been used to measure the deflection of non-piezoelectric cantilevers ${ }^{37,38}$. In this paper, we suggest to use PVDF as measurement of the deflection in a cantilever piezoelectric actuator. The advantages relative to the measurement in ${ }^{25}$ described above is that the electrodes surfaces of the sensing and of the actuation are much larger and thus the sensing sensintivity as well as the actuation performances are much better. Relative to self-sensing techniques, also described above, there are no couplings between the actuation and the sensing because their electrodes are completely different. This makes the conditioning (electrical circuit, modeling, observer) easier: the actuation powering is not coupled with the sensing electrical circuit and conditioning. Whilst generalizable into multi-layers cantilever, we focus in this paper on a three-layers structure that we call actuator with collocated sensor: two external piezoelectric layers are used for the actuation and one middle piezoelectric layer is used for the sensing.

The rest of the paper is organized as follows. In section. 2, we present the principle of the three-layerered actuator with collocated sensor. Section. 3 is devoted to the modeling and the observer derivation that permits to estimate the displacement and the force. In section. 4, the simulation results are presented. Finally some conclusions and future works are given in Section. 5.

\section{PRINCIPLE OF THE THREE-LAYERS ACTUATOR WITH COLLOCATED SENSOR}

\subsection{General principle}

The structure is a cantilever composed of three principal piezoelectric layers (Fig. 1-a). The upper layer as well as the lower layer serve for the actuation and the middle layer serves for the sensing. In order to avoid interferences between the electrodes of the sensing layer and the actuation layers, a very thin electrical insulator is placed between the middle layer and each of the external layers. In fact, this insulator could be an insulating glue that is used to bond the different layers. The actuation layers should have a stiffness much larger than that of the sensing layer. For that, a lead zirconate titanate (PZT) piezoelectric material is suggested for the actuation whilst a PVDF piezoelectric polymer for the sensing. In order to make the study more general, we study the case where the thickness of the upper actuation layer (denoted $h_{a h}$ ) is different from the thickness of the lower actuation layer (denoted $h_{a l}$ ) (Fig. 1-b). Fig. 1-c depicts the CAD design of the three-layers structure before and after assembly. The cantilever is glued on a support and we assume that the fixation behaves like a clamping. The extremity of the cantilever in a free space above the support is split into three parts such that 
we can easily extract the electrodes of the three layers. Let Tab. 1 depict the different geometrical and physical properties. In this, we do not consider the glue and will assume that its thickness is negligible relative to $h_{a h}$, to $h_{a l}$ and to $h_{s}$ and its stiffness is also negligible relative to those of the three piezoelectric layers. The numerical values of the different parameters for the materials are also reported in the table: PZT for the actuation layers and PVDF for the sensing layer. Some of these values will be used for the further simulation. The width, the thicknesses and the length will be varied during the simulation.
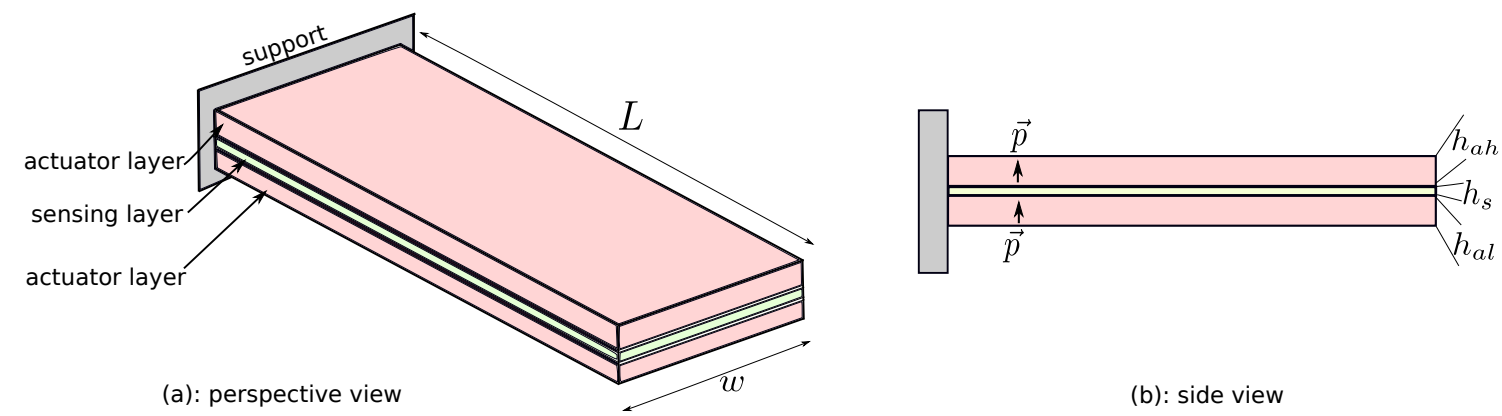

(a): perspective view

parts for electrical
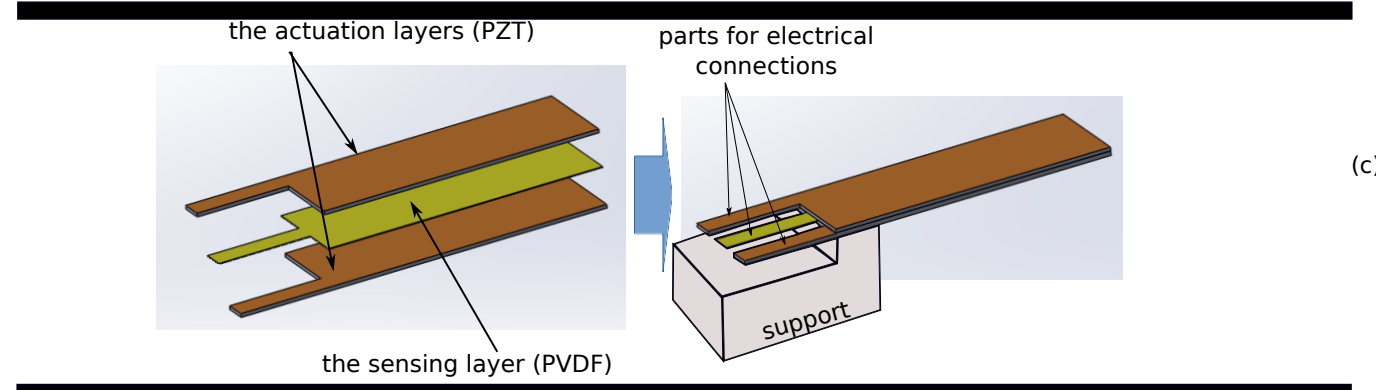

(b): side view

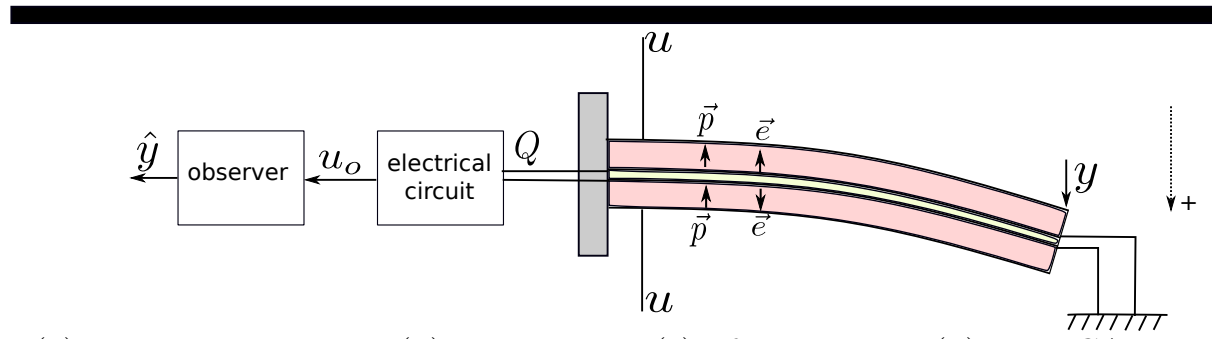

(c)

(d)

Figure 1: (a): perspective view. (b): side view. (c): functioning. (d): the CAD design of the three-layers structure.

The functioning of the actuation is as follows. The two actuation piezoelectric layers (upper and lower) have the same poling $\vec{p}$ direction, as indicated in Fig. 1-b. Let the internal electrodes of these two layers be the ground. When a positive potential $u$ is applied to the upper electrode of the upper layer, the electric field $\vec{e}$ appearing in this latter is in the same direction than $p$ and consequently it expands. If the same potential $u$ is applied to the lower electrode of the lower layer, the electric field $\vec{e}$ is in the opposite direction than $p$ and consequently this layer contracts. This expansion and contraction of the above and of the lower part of the cantilever results in a global bending $y$ of this latter as illustrated in Fig. 1-d. A negative bending of $y$ is therefore obtained by applying a negative potential $u$. In the sequel, the voltage $u=u[V]-0 V$ (potentials difference) will be used. Remind that the expansion and contraction of the layers resulting from the electrical field $\vec{e}$ is thanks to the converse piezoelectric effect and is quantified by the transverse piezoelectric constant $d_{31}$.

The functioning of the sensing is as follows. During the bending of the cantilever, the middle layer is subjected to the same bending $y$ (that we will call displacement) and consequently to internal stress. Due to this stress, 
Table 1: Geometrical and physical properties.

\begin{tabular}{|c|c|c|}
\hline signification & symbol & value \\
\hline length of the cantilever & L & \\
\hline width of the cantilever & $w$ & \\
\hline thickness of the upper actuation layer & 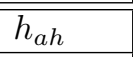 & \\
\hline transversal piezoelectric constant of the upper actuation layer (PZT) & $d_{31 a}$ & $-320 \times 10^{-12} \mathrm{~m} / \mathrm{V}$ \\
\hline axial piezoelectric constant of the upper actuation layer (PZT) & $d_{33 a}$ & $593 \times 10^{-12} \mathrm{~m} / \mathrm{V}$ \\
\hline transversal piezoelectric stress constant of the upper actuation layer (PZT) & $g_{31 a}$ & $10 \times 10^{-3} V m / N$ \\
\hline longitudinal piezoelectric stress constant of the upper actuation layer (PZT) & $g_{33 a}$ & $-10 \times 10^{-3} \mathrm{Vm} / \mathrm{N}$ \\
\hline compliance of the upper actuation layer (PZT) & $s_{11 a}$ & $16 \times 10^{-12} \mathrm{~m}^{2} / \mathrm{N}$ \\
\hline Youngs modulus of the upper actuation layer (PZT) & $E_{a}$ & $115 \times 10^{9} \mathrm{~N} / \mathrm{m}^{2}$ \\
\hline density (PZT) & $\rho_{a}$ & $7800 \mathrm{~kg} / \mathrm{m}^{3}$ \\
\hline thickness of the lower actuation layer & $\overline{h_{a l}}$ & \\
\hline transversal piezoelectric constant of the lower actuation layer (PZT) & $d_{31 a}$ & $-320 \times 10^{-12} \mathrm{~m} / \mathrm{V}$ \\
\hline axial piezoelectric constant of the lower actuation layer (PZT) & $d_{33 a}$ & $593 \times 10^{-12} \mathrm{~m} / \mathrm{V}$ \\
\hline transversal piezoelectric stress constant of the lower actuation layer (PZT) & $g_{31 a}$ & $10 \times 10^{-3} V m / N$ \\
\hline longitudinal piezoelectric stress constant of the lower actuation layer (PZT) & $g_{33 a}$ & $-10 \times 10^{-3} \mathrm{Vm} / \mathrm{N}$ \\
\hline compliance of the lower actuation layer (PZT) & $s_{11 a}$ & $16 \times 10^{-12} \mathrm{~m}^{2} / N$ \\
\hline Youngs modulus of the lower actuation layer (PZT) & $E_{a}$ & $115 \times 10^{9} \mathrm{~N} / \mathrm{m}^{2}$ \\
\hline density (PZT) & $\rho_{a}$ & $7800 \mathrm{~kg} / \mathrm{m}^{3}$ \\
\hline thickness of the sensing layer & 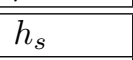 & \\
\hline transversal piezoelectric constant of the sensing layer (PVDF) & $d_{31 s}$ & $20 \times 10^{-12} \mathrm{~m} / \mathrm{V}$ \\
\hline axial piezoelectric constant of the sensing layer (PVDF) & $d_{33 s}$ & $-33 \times 10^{-12} \mathrm{~m} / \mathrm{V}$ \\
\hline transversal piezoelectric stress constant of the sensing layer (PVDF) & $g_{31 s}$ & $216 \times 10^{-3} V m / N$ \\
\hline longitudinal piezoelectric stress constant of the sensing layer (PVDF) & $g_{33 s}$ & $-330 \times 10^{-3} V m / N$ \\
\hline compliance of the sensing layer (PVDF) & $s_{11 s}$ & $3.65 \times 10^{-10} \mathrm{~m}^{2} / \mathrm{N}$ \\
\hline Youngs modulus of the sensing layer (PVDF) & $E_{s}$ & $3 \times 10^{9} \mathrm{~N} / \mathrm{m}^{2}$ \\
\hline density (PVDF) & $\rho_{s}$ & $1780 \mathrm{~kg} / \mathrm{m}^{3}$ \\
\hline total thickness $\left(h=h_{a h}+h_{a l}+h_{s}\right)$ & 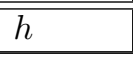 & \\
\hline
\end{tabular}

electric charge $Q$ appears on the electrodes of the layer thanks to the direct piezoelectric effect. This charge can be amplified and transformed by an electrical circuit into an exploitable voltage $u_{o}$. An algorithm that we call observer and which is based on the model of the electrical circuit and on the model of the piezoelectric layer can therefore be used to provide an estimate $\hat{y}$ of the real displacement $y$.

\subsection{The electrical circuit}

The objective of the electrical circuit is to transform the charge $Q$ into an exploitable voltage $u_{o}$. There are different circuit schemes ${ }^{30,39,42}$. In this paper we suggest the classical charge amplifier. In this, we consider the equivalent capacitor $C_{c}$ and equivalent resistor $R_{c}$ of the cable as suggested in ${ }^{39}$. Fig. 2-a illustrates therefore the electrical circuit.

In the sequel, we will consider that the operational amplifier (op-amp) is not ideal and thus a bias current $i_{\text {bias }}$ occurs in it. We will also consider that the sensing piezoelectric layer has an internal resistor $R_{p}$ that causes a charge internal leakage. consequently, the recuperated charge $Q$ is not anymore equal to the charge generated by the direct piezoelectric effect, that we call $Q_{p}$ in the sequel, but is less. The different amount of charge will be formulated in section. 3.2. Fig. 2-b depicts therefore the equivalent circuit of the piezoelectric layer, where $C_{p}$ is its equivalent capacitor. 


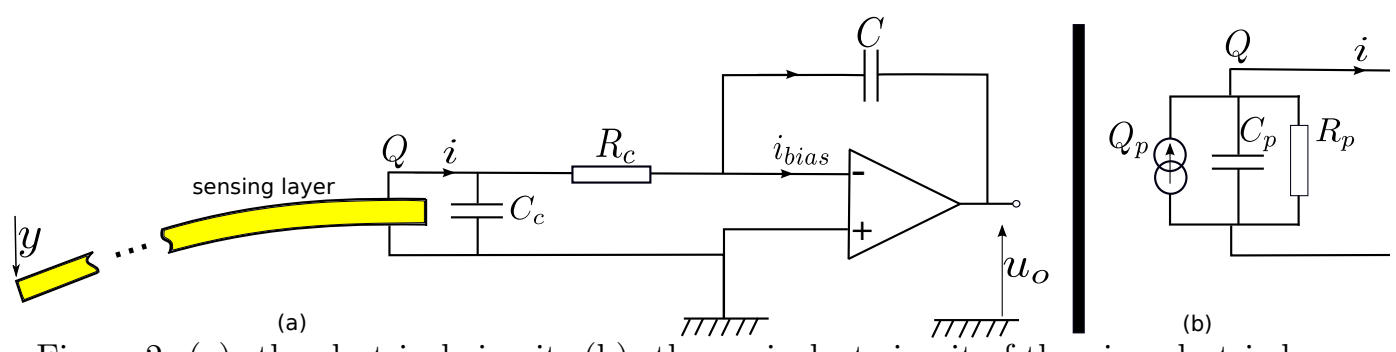

Figure 2: (a): the electrical circuit. (b): the equivalent circuit of the piezoelectric layer.

\section{MODELING AND OBSERVER}

First we model the actuation behavior of the cantilever that relates the applied voltage $u$ with the resulting displacement $y$. Then, we model the sensing behavior as well as the electrical circuit for the sensing. Finally, from these sensing behavior and circuit models, we derive the observer that permits to provide the estimation of the displacement.

\subsection{Modeling the actuation behavior}

In ${ }^{40}$, the static model of a multilayered piezoelectric cantilever actuator has been established. The model is linear and the layers can be active (piezoelectric) or passive (non-piezoelectric). In our case, the sensing layer is passive with regard to the actuation. Thus, we have a three layer cantilever with two external active layers and one middle passive one. Using the general form from ${ }^{40}$, we derive the actuation model of our cantilever:

$$
y=\frac{m_{\text {piezo }} L}{D} u
$$

where:

$$
m_{\text {piezo }}=\frac{w}{2}\left(\frac{d_{31 a}}{s_{11 a}}\left[4 \bar{z}-3 h_{a h}-2 h_{s}-h_{a l}\right]+\frac{d_{31 s}}{s_{11 s}}\left[2 \bar{z}-2 h_{a h-h_{s}}\right]\right)
$$

and

$$
D=\frac{w}{3}\left[D_{1}+D_{2}+D_{3}\right]
$$

with:

$$
\left\{\begin{array}{l}
D_{1}=\frac{h_{a h}}{s_{11 a}}\left[3\left(\bar{z}-h_{a h}\right) \bar{z}+h_{a h}^{2}\right] \\
D_{2}=\frac{h_{s}}{s_{11 s}}\left[3\left(\bar{z}-h_{a h}-h_{s}\right)\left(\bar{z}-h_{a h}\right)+h_{s}^{2}\right] \\
D_{3}=\frac{h_{a l}}{s_{11 a}}\left[3\left(\bar{z}-h_{a h}-h_{s}-h_{a l}\right)\left(\bar{z}-h_{a h}-h_{s}\right)+h_{a l}^{2}\right]
\end{array}\right.
$$

and with $\bar{z}$ being distance of the neutral axis from the bottom surface of the cantilever and is given by:

$$
\bar{z}=\frac{-\left[\left(\frac{h_{a h}^{2}}{s_{11 a}}+\frac{h_{s}^{2}}{s_{11 s}}+\frac{h_{a l}^{2}}{s_{11 a}}\right)-2\left(\frac{h_{a h}^{2}}{s_{11 a}}+\frac{h_{s}\left(h_{a h}+h_{s}\right)}{s_{11 s}}+\frac{h_{a l}\left(h_{a h}+h_{s}+h_{a l}\right)}{s_{11 a}}\right)\right]}{2\left(\frac{h_{a h}}{s_{11 a}}+\frac{h_{s}}{s_{11 s}}+\frac{h_{a l}}{s_{11 a}}\right)}
$$

The model in Eq. 1 is the static behavior of the three-layer cantilever. This can be used to have an idea of its static performances, more precisely its range of actuation for a given range of voltage. In order to have an idea of its dynamics, the resonant frequency can also be given. The first resonant frequency of the three-layer structure is derived from the resonant frequency of a multilayer structure in ${ }^{40}$. We obtain: 


$$
f_{r}=\frac{1.8751}{2 \pi L^{2}} \sqrt{\frac{D}{\mu}}
$$

where $\mu$ is the linear mass, with $\mu=\frac{m_{c}}{L}=w\left(\rho_{a} h_{a h}+\rho_{s} h_{s}+\rho_{a} h_{a l}\right), m_{c}$ being the mass of the whole cantilever.

It is worthy to notice that the above static and frequency properties can be used to design the actuator in order to find appropriate dimensions for given specified performances, or conversely, to have an estimate performances of a given three-layers cantilever.

\subsection{Modeling the sensing behavior}

The sensing part is composed of the PVDF layer and the electrical circuit. In this subsection, we model both parts.

\subsubsection{The PVDF layer}

PVDF polymer, which is the material for the sensing layer, has a piezoelectricity property initially observed in 41. It is widely used as sensors in various applications: medical, microphones, military, industrial, vibrations, micromanipulation, $\ldots{ }^{42-45}$ thanks to several reasons. First, it has a higher sensitivity relative to piezoceramics as it can generate voltage 10-25 times higher ${ }^{43}$. PVDF is also widely available in sellers and has a low cost. Finally, PVDF has a very high flexibility making it easy to integrate inside an existing structure without modifying the mechanical properties of this latter. Consequently a PVDF sensor can generally track the dynamics of the mechanical structure on which it is placed.

During the bending of the cantilever due to the actuation, let us assume that there is a uniform pressure $P$ (in $\left.\left[\frac{N}{m}\right]\right)$ along the entire length $L$ width which is caused by the internal stress. This uniform pressure $P$ is directly found at the sensor layer. From the general model of multilayer in ${ }^{40}$, the relation between the displacement $y$ and the pressure load $P$ for a three-layers cantilever is derived:

$$
y=\frac{w L^{4}}{8 D} P
$$

where $D$ is the coefficient defined in the previous subsection.

On the other hand, the generated charge $Q_{p}$ due to the uniform pressure $P$ applied to the three-layer structure is also derived from ${ }^{40}$ :

$$
Q_{p}=\frac{w m_{\text {piezo-s }} L^{3}}{6 D} P
$$

where $m_{\text {piezo-s }}$ is the coefficient for the sensing layer and is defined as follows:

$$
m_{\text {piezo-s }}=\frac{w d_{31 s}}{2 s_{11 s}}\left[6 \bar{z}-5 h_{a h}-3 h_{s}-h_{a l}\right]
$$

The formulae of the charge $Q_{p}$ in Eq. 8 provides the total charge from three layers, all based on PVDF materials. However, in our case, only the middle layer furnishes the charge, and remind that the two external layers are PZT. We therefore yield the charge $Q_{p}$ from one layer of the three-layers structure:

$$
Q_{p}=\frac{w m_{\text {piezo-s }} L^{3}}{18 D} P
$$

Combining Eq. 10 with Eq. 7, we obtain:

$$
Q_{p}(t)=\frac{4 m_{\text {piezo-s }}}{9 L} y(t)=d_{p} y(t)
$$


where $d_{p}=\frac{4 m_{\text {piezo-s }}}{9 L}$ is called displacement-to-charge coefficient. It is also worthy to notice that, because of the high flexibility of the PVDF layer, it does not modify (in practice) the behavior of the actuation and it can track the dynamics of this latter.

Due to the possible internal leakage within the PVDF layer however, the whole recuperated charge $Q_{p}$ does not go to the electrical circuit. Remind that $R_{p}$ is the internal resistor and $C_{p}$ is the equivalent capacitor of the PVDF layer (Fig. 2-b). Thus, the available charge $Q(t)$ for the electrical circuit is:

$$
Q(t)=Q_{p}(t)-\frac{U_{p}}{R_{p}}=Q_{p}-\frac{Q_{p}}{C_{p} R_{p}}=Q_{p}\left(1-\frac{1}{R_{p} C_{p}}\right)=d_{p}\left(1-\frac{1}{R_{p} C_{p}}\right) y(t)
$$

in which $U_{p}=\frac{Q_{p}(t)}{C_{p}}$ is the voltage across the PVDF layer due to the generated charge.

\subsubsection{The electrical circuit and the output voltage}

The electrical scheme in Fig. 2-a which evidences the bias current in the op-amp permits to yield:

$$
\frac{d Q(t)}{d t}=i_{\text {bias }}-C \frac{d u_{o}(t)}{d t}
$$

Notice that $i_{\text {bias }}$ could vary over the time but at a low rate. However, we assume it to be constant.

Combining Eq. 13 with Eq. 12, the output voltage $u_{o}(t)$ is:

$$
u_{o}(t)=\frac{1}{C} i_{\text {bias }}-\frac{d_{p}}{C}\left(1-\frac{1}{R_{p} C_{p}}\right) y(t)
$$

We can observe from Eq. 14 that the exploitable voltage $u_{o}(t)$ is affine relative to the displacement $y(t)$ and linear if $i_{\text {bias }}=0 \mathrm{~A}$. Furthermore, the relation is static. This means that there is no intermediary dynamics between the displacement $y$ and the exploitable voltage $u_{o}$ and consequently it is theoretically possible to follow the dynamics of the actuator by the suggested sensing method.

\subsection{The observer}

The role of the observer is to provide an estimate $\hat{y}(t)$ of the real displacement $y(t)$ from the sole available signal, i.e. from the exploitable voltage $u_{o}(t)$. It is an algorithm based on the model of the structure and on the model of the electrical circuit. From Eq. 14, we directly derive the observer as follows:

$$
\hat{y}(t)=\frac{R_{p} C_{p}}{d_{p}\left(R_{p} C_{p}-1\right)}\left(i_{\text {bias }}-C u_{o}(t)\right)
$$

\section{SIMULATION RESULTS}

In this section, we simulate the behavior of the three-layers cantilever. First we simulate the actuation functioning with different geometrical conditions. Then we simulate the sensing functioning in order to have an idea of its performances. 


\subsection{Actuation functioning}

The model developed in section. 3.1 is used here. First the displacement $y$ is evaluated when the thicknesses of the actuation layers are varied. For that, we impose a voltage $u=50 \mathrm{~V}$ and we suppose that the two thicknesses are similar: $h_{a l}=h_{a h}$. Furthermore, the width is set $w=1 \mathrm{~mm}$. Fig. 3 -a depicts the results when the thickness $h_{a l}=h_{a h}$ is varied from $50 \mu \mathrm{m}$ to $250 \mu \mathrm{m}$. As we can observe, the displacement decreases when the thickness increases. This is due to the stiffness of the cantilever which augments with the increase of the two thicknesses. We also deduce that for cantilever actuators of which the PZT thickness is $100 \mu m, 150 \mu m$ and $200 \mu m$, the results come back to the results in classically sized bimorph actuators (for in instance in ${ }^{5,6}$ ) which use standard and commercially available thickness of PZT. When the PZT thickness is lower, much less than $100 \mu m$, the displacement is very high. However, in this case, the stiffness of the actuator is also reduced and thus its force capability (blocking force) also decreases. Furthermore, since such thickness is nowadays not standard and not commercially available, one should perform PZT layer thinning, for instance in clean-room ${ }^{47}$. Notice that, from the results in Fig. 3-a, the thickness $h_{s}$ of the sensing layer has a very small effect on the performance of the actuator. Once again, this is thanks to the high compliance of PVDF material. Fig. 3-b depicts the evolution of the resonant frequency of the cantilever actuator over the thickness $h_{a h}=h_{a l}$ which illustrates the linear increase. Once again, we observe the same frequencies of existing bimorph actuators ${ }^{5,6}$ when the thickness is standard and commercially available $(100 \mu \mathrm{m}, 150 \mu \mathrm{m}, 200 \mu \mathrm{m}$ and $250 \mu \mathrm{m})$.

The next simulation deals with the study of the output displacement $y$ versus the length $L$ of the cantilever. The simulation is still carried out with a voltage $u=50 \mathrm{~V}$, a width $w=1 \mathrm{~mm}$ and a sensing layer thickness $h_{s}=50 \mu \mathrm{m}$. The results are pictured in Fig. 3 -c which confirm the increase of the tip displacement with the length. The performance difference between a PZT layer of $h_{a h}=h_{a l}=200 \mu m$ and that of $h_{a h}=h_{a l}=100 \mu m$ is also distinctly evidenced. This clearly confirms the interest of having smaller thickness of actuation layer. In fact, beyond the stiffness reason, reducing the actuation layer thickness increases the electrical field (for the same given voltage) and thus will increase the deformation. Fig. 3-d presents the resonant frequency versus the length which shows its exponential increase when reducing this latter. A compromise between the wanted output displacement and the resonant frequency should therefore be done when choosing the length. Furthermore, the length is an important parameter of miniaturization which can be essential during a design step.

Finally we check the effect of the width $w$ on the performances. Fig. 3-e and $\mathrm{f}$ shows the output displacement when applying $u=50 \mathrm{~V}$ and the resonant frequency respectively with different conditions of thicknesses, the length being left $L=100 \mu m$ They demonstrate that the width does not affect these performances. The reasons are that the width does not affect neither the electrical field within the layers nor the stiffness of the cantilever. A cantilever with a large width is similar to two cantilevers with smaller widths and placed side by side. These two cantilevers have the same performances, and also have the same performances than the large cantilever.

\subsection{Sensing functioning}

The model developed in section. 3.2 is used here. First, we observe the displacement-to-charge coefficient $d_{p}$ for different conditions. Fig. 4-a depicts the results when we vary $w$. As we can see, $d_{p}$ linearly increases (in absolute value) with the width. This is thanks to the increase of the electrodes surface. We now study the evolution of $d_{p}$ when the length is increased. In this case, the electrodes surface also increases and consequently so is the amount of charge. However, as the output displacement $y$ at the tip also augments with a the $L$, the final displacement-to-charge ratio does not evolve anymore, or even decreases in absolute value, as illustrated in Fig. 4-b.

Now, in order to verify the exploitable voltage $u_{o}$, we perform simulation with fixed geometrical values: $h_{s}=50 \mu \mathrm{m}, h_{a h}=h_{a l}=200 \mu \mathrm{m}, L=20 \mathrm{~mm}$ and $w=2 \mathrm{~mm}$. For the electrical circuit and piezoelectric equivalence, we use the following numerical values taken in part from ${ }^{30}: C=4.7 p F, C_{p}=8.2 n F, R_{p}=437 G \Omega$ and $i_{\text {bias }}=-1.7 \times 10^{-12} \mathrm{~A}$. When the actuator is supplied by a voltage $u= \pm 100 \mathrm{~V}$ which yields a displacement $y= \pm 80 \mu m$, the exploitable voltage from the sensing layer and the electrical circuit is depicted in Fig. 4 . It shows that $u_{o}$ ranges between values that can be easily amplified or exploited: from $-401 \mathrm{mV}$ to $-310 \mathrm{mV}$. A well designed conditioner can amplify and shift this range before sending the signal to the observer. As a conclusion the sensing methodology is very promising in term of implementability and realizability. 


\section{CONCLUSIONS}

This paper presents a piezoelectric actuator with an embedded piezoelectric sensor for micromanipulation and microassembly tasks. The whole system has a three-layers cantilever structure. The two external layers which are based on the PZT material serve for the actuation and the middle layer which is based on the PVDF material serves for the sensing. An electrical circuit is used to extract the charges from the sensing layer. The actuation behavior and the sensing functioning, with the electrical circuit, are successively modeled. Then an observer was derived from the sensing model in order to provide an estimate measure of the actuator displacement. Extensive simulation is carried out and discussed, and the different results show the significance of the new actuator with collocated sensors in precise positioning. The results particularly show that the sensing element does not affect the performances of the actuation and can fully track its performances. Future works include the fabrication of the design and the experimental characterizations in order to validate its principle and its model. Future works also include the use of methodologies of design such as in ${ }^{48,49}$ in order to optimize the structure dimensions and the performances. These designs are based on control theory tools such as interval techniques ${ }^{50}$
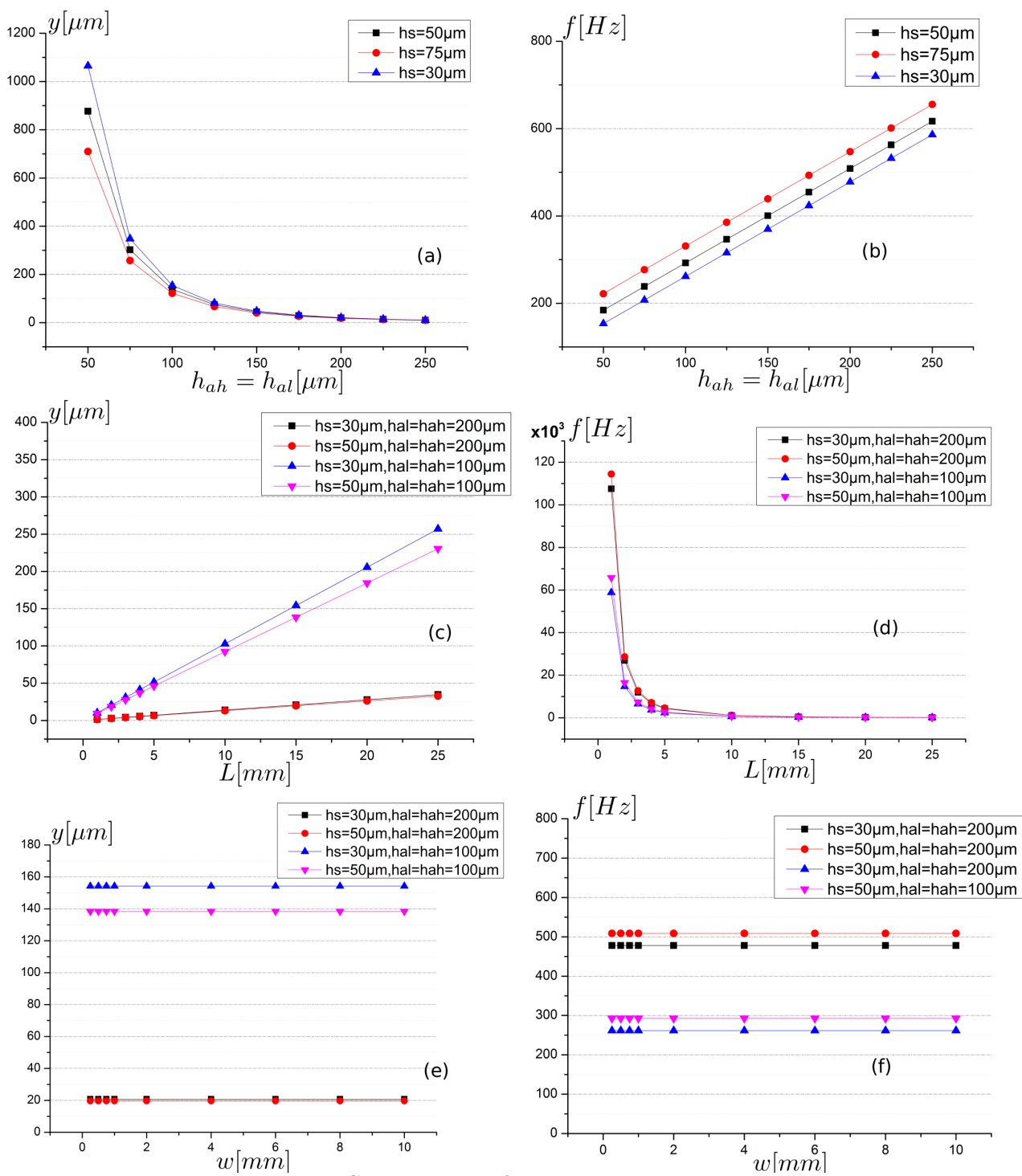

Figure 3: Simulation of the actuation behavior. 

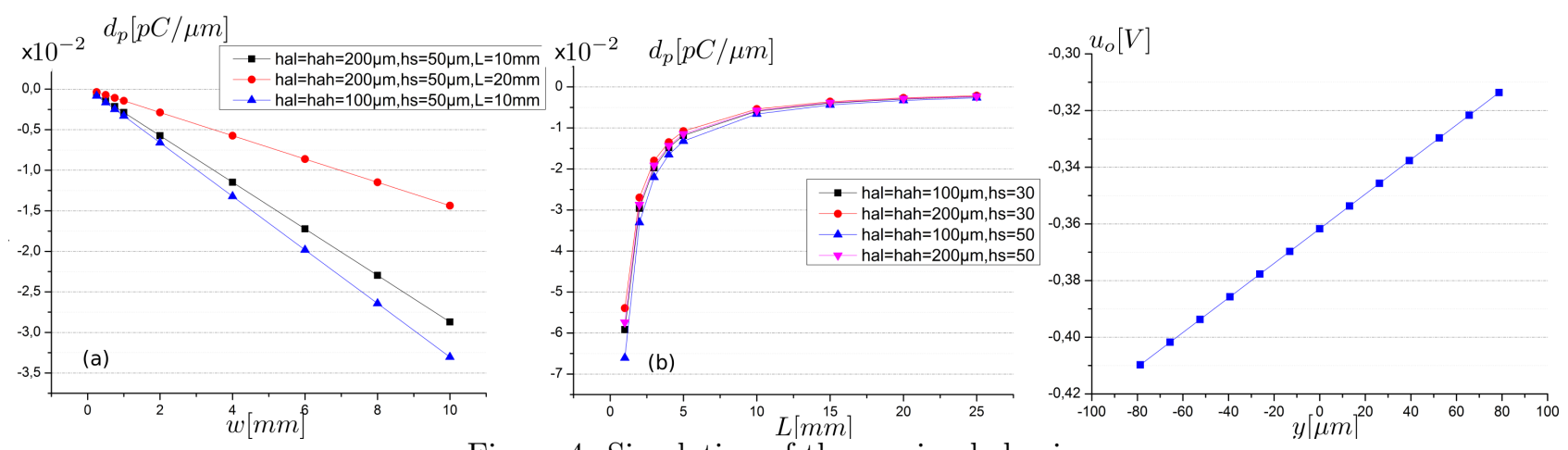

Figure 4: Simulation of the sensing behavior.

\section{ACKNOWLEDGMENTS}

This work is supported by the national ANR-JCJC C-MUMS-project (National young investigator project ANR12-JS03007.01: Control of Multivariable Piezoelectric Microsystems with Minimization of Sensors). This work is also supported by the LABEX ACTION (ANR-11- LABX-0001-01)..

\section{REFERENCES}

[1] J. Agnus, N. Chaillet, C. Clévy, S. Dembélé, M. Gauthier, Y. Haddab, G. Laurent, P. Lutz, N. Piat, K. Rabenorosoa, M. Rakotondrabe and B. Tamadazte, "Robotic microassembly and micromanipulation at FEMTO-ST," Journal of Micro-Bio Robotics, vol. 8, no. 2, pp. 91-106, 2013.

[2] M. Rakotondrabe, Smart materials-based actuators at the micro/nano-scale: characterization, control and applications, Springer-Verlag, NewYork, 2013.

[3] S. Devasia, E. E. Eleftheriou, R. Moheimani, "A survey of control issues in nanopositioning", IEEE Transactions on Control Systems Technology, Vol.15, Nº , pp.802-823, 2007.

[4] M. Rakotondrabe, 'Piezoelectric systems for precise and high dynamic positioning: design, modeling, estimation and control', HDR halititation thesis, University of Franche-Comté / FEMTO-ST, November 10, 2014.

[5] Y. Haddab, N. Chaillet, A. Bourjault, "A microgripper using smart piezoelectric actuators", IEEE International Conference on Intelligent Robots and Systems, 2000.

[6] J. Agnus, P. Nectoux, N. Chaillet, "Overview of microgrippers and design of a micromanipulation station based on a MMOC microgripper", IEEE International Symposium on Computational Intelligence in Robotics and Automation, 2005.

[7] M. Rakotondrabe and A. Ivan, 'Development and Force/Position Control of a New Hybrid ThermoPiezoelectric microGripper dedicated to micromanipulation tasks', IEEE Transactions on Automation Science and Engineering, 8(4), pp.824-834, 0ctober 2011.

[8] S. Khadraoui, M. Rakotondrabe and P. Lutz, 'Interval force/position modeling and control of a microgripper composed of two collaborative piezoelectric actuators and its automation', International Journal of Control, Automation and Systems, 12(2), Page 358-371, April 2014.

[9] M. Rakotondrabe, C. Clévy and P. Lutz, 'Modelling and robust position/force control of a piezoelectric microgripper', IEEE International Conference on Automation Science and Engineering, Scottsdale AZ USA, 2007.

[10] C. Clévy, M. Rakotondrabe and N. Chaillet, 'Signal measurement and estimation techniques issues in the micro/nano world', edited book, Springer - Verlag, New York, ISBN 978-1-4419-9945-0, August 2011.

[11] F. Arai, A. Kawaji, T. Sugiyama, Y. Onomura, M. Ogawa, T. Fukuda, H. Iwata and K. Itoigawa, "3d micromanipulation system under microscope", IEEE International Symposium on Micromechatronics and Human Science, 1998. 
[12] M. Goldfarb and N. Celanovic, "A flexure-based gripper for small-scale manipulation", Robotica, 17, 1999.

[13] Y. Haddab, Q. Chen and P. Lutz, "Improvement of strain gauges micro-forces measurement using Kalman optimal filtering", Mechatronics, 19(4), 2009.

[14] D. Croft, G. Shed and S. Devasia, "Creep, hysteresis and vibration compensation for piezoactuators: atomic force microscopy application", ASME Journal of Dynamic Systems, Measurement and Control, 2001.

[15] M. Rakotondrabe, C. Clévy and P. Lutz, 'Complete open loop control of hysteretic, creeped and oscillating piezoelectric cantilever', IEEE Transactions on Automation Science and Engineering, Vol.7(3), pp:440-450, July 2010.

[16] D. Habineza, M. Rakotondrabe and Y. Le Gorrec, ' Simultaneous Suppression of Badly-Damped Vibrations and Cross-couplings in a 2-DoF piezoelectric actuator, by using Feedforward Standard H-inf approach', SPIE Sensing Technology+Applications; Sensors for Next Generation Robots conference , 9494-29, Baltimore Maryland USA, April 2015.

[17] G. Schitter, A. Stemmer, F. Allgower, "Robust 2 DOF-control of a piezoelectric tube scanner for high speed atomic force microscopy", American Control Conference, pp. 3720-3725, June 2003.

[18] M. Al Janaideh, M. Rakotondrabe and O. Al Janaideh, 'Further Results on Hysteresis Compensation of Smart Micro-Positioning Systems with the Inverse Prandtl-Ishlinskii Compensator', IEEE Transactions on Control Systems Technology, doi:10.1109/TCST.2015.2446959, 2016.

[19] O. Aljanaideh, D. Habineza, M. Rakotondrabe and M. Al Janaideh, 'Experimental comparison of ratedependent hysteresis models in characterizing and compensating hysteresis of piezoelectric tube actuators', Elsevier Physica B: Condensed Matter, doi:10.1016/j.physb.2015.10.021, oct 2015.

[20] W. T. Ang, P. K. Kholsa and C. N. Riviere, Feedforward controller with inverse rate-dependent model for piezoelectric actuators in trajectory-tracking applications, IEEE/ASME Transactions on Mecha- tronics, Vol.12(2), pages 134-142, April 2007.

[21] Y. Al Hamidi and M. Rakotondrabe, 'Multi-Mode Vibration Suppression in 2-DOF Piezoelectric Systems Using Zero Placement Input Shaping Technique', SPIE Sensing Technology+Applications; Sensors for Next Generation Robots conference, 9494-27, Baltimore Maryland USA, April 2015.

[22] D. Habineza, M. Rakotondrabe and Y. Le Gorrec, 'Bouc-Wen Modeling and Feedforward Control of multivariable Hysteresis in Piezoelectric Systems: Application to a 3-DoF Piezotube scanner', IEEE Transactions on Control Systems Technology, Vol 23, Issue 5, Page 1797-1806, Sept 2015.

[23] M. Rakotondrabe, 'Modeling and Compensation of Multivariable Creep in multi-DOF Piezoelectric Actuators', IEEE International Conference on Robotics and Automation, pp.4577-4581, St Paul Minnesota USA, May 2012.

[24] M. Rakotondrabe, 'Classical Prandtl-Ishlinskii modeling and inverse multiplicative structure to compensate hysteresis in piezoactuators', American Control Conference, pp.1646-1651, Montral Canada, June 2012.

[25] D. Campolo, R. Sahai and R.S. Fearnig, 'Development of piezoelectric bending actuators with embedded piezoelectric sensors for micromechanical flapping mechanisms', IEEE ICRA, Taipei, Taiwan 2003.

[26] J.J. Dosch, D.J. Inman and E. Garcia, "A Self-Sensing Piezoelectric Actuator for Collocated Control", Journal of Intell. Mater. Syst. and Struct., vol 3, pp. 166-185, 1992.

[27] T. Takigami, K. Oshima, Y. Hayakawa and M. Ito, "Application of self-sensing actuator to control of a soft-handling gripper", Proc. to IEEE ICCA, pp. 902-906, Italy, 1998. x

[28] Y. Cui, Self-Sensing, "Compounding Control of Piezoceramic Micro-Motion Worktable Based on Integrator", Proc. to 6th World Congress on Intell. Cont. and Autom., China, 2006.

[29] A. S. Putra, H. Sunan, T. K. Kok, S.K. Panda and T. H. Lee, Self-sensing actuation with adaptive control in applications with switching trajectory, IEEE/ASME Trans. on Mechatronics, Vol.13(1), pp.104-110, 2008.

[30] A. Ivan, M. Rakotondrabe, P. Lutz and N. Chaillet, "Quasi-static displacement self-sensing method for cantilevered piezoelectric actuators", Review of Scient. Instr., Vol.80(6), 065102, June 2009.

[31] A. Ivan, M. Rakotondrabe, P. Lutz and N. Chaillet, "Current integration force and displacement self-sensing method for cantilevered piezoelectric actuators", Review of Scient. Instr., Vol.80(12), 2126103, Dec 2009.

[32] M. Rakotondrabe, A. Ivan, S. Khadraoui, C. Clévy, P. Lutz and N. Chaillet, "Dynamic displacement self-sensing and robust control of cantilevered piezoelectric actuators dedicated to microassembly tasks", IEEE/ASME Inter. Conf. on Intelligent Mechatronics, pp:557-562, Canada, July 2010. 
[33] M. Rakotondrabe, A. Ivan, S. Khadraoui, P. Lutz and N. Chaillet, 'Simultaneous displacement and force self-sensing in piezoelectric actuators and applications to robust control of the displacement', IEEE/ASME Transactions on Mechatronics, Vol 20, No 2, Page 519 - 531, April 2015.

[34] A. Ivan, J. Agnus and M. Rakotondrabe, 'Micropositioning device with multidegrees of freedom for piezoelectric actuators and associated method ', PCT US patent, US20150054520 A1, USA extension of the frenche patent, INPI FR-N12/52554, delivered in march 2013.

[35] Y. Ishikiriyama and T. Morita, "Improvement of self-sensing piezoelectric actuator control using permittivity change detection", Journal of Advanced Mechanical Design, Systems, and Manufacturing,Vol.4, 2010.

[36] K. Kundert, "Modeling dielectric absorption in capacitors", The designer's guide community, pp.1-19, 2008.

[37] D.H. Kim, D. Kim, S.M. Kim and H. Kang, "Development of a piezoelectric polymer-based sensorized microgripper for microassembly and micromanipulation", IROS, 2003.

[38] Y. Shen, N. Xi, C.A. Poumeroy, U.C. Wejinya and W.J. Li, "An active micro-force sensing system with piezoelectric servomechanism", IROS, Barcelona, Spain, April 2005.

[39] J. Karki, 'Signal Conditioning Piezoelectric Sensors', Texas Instrument, Application report SLOA033A, Sept 2003.

[40] R. G. Ballas, 'Piezoelectric multilayer beam bending actuators: static and dynamic behavior and aspects of sensor integration', Springer-Verlag, Berlin 2007.

[41] H. Kawai, "The piezoelectricity of poly (vinylidene fluoride)", JPN. J. Appl. Phys. 8, pp. 975-976, 1979.

[42] A.V. Shirinov and W.K. Schomburg, "Pressure sensor from a PVDF film", Elsevier Sensors and Actuators A: Physical, 142, 4855, 2008.

[43] J. Xu, M.J. Dapino, D. Gallego-Perez and D. Hansford, "Microphone based on Polyvinylidene Fluoride (PVDF) micro-pillars and patterned electrodes", Sensors and Actuators A: Physical, 153, 24-32, 2009.

[44] B. Bao, D. Matthews, A. Munyard, H. Sun and J. Pan, "Visualization of the surface vibrating using a PVDF film array", Acoustic, Nov 2015.

[45] T. Sharma, S-S. Je, B. Gill and J.X.J. Zhang, "Patterning piezoelectric thin film PVDFTrFE based pressure sensor for catheter application", Sensors and Actuators A: Physical, 177, 87-92, 2012.

[46] M. Eastman, "Smart Sensors Based On Piezoelectric PVDF", Department of Chemistry, The University of Texas at El Paso, 2015.

[47] A. Bienaimé, V. Chalvet, C. Clévy, L. Manuel-Gauthier, T. Baron and M. Rakotondrabe, 'Static / dynamic trade-off performance of PZT thickfilm micro-actuators', IOP Journal of Micromechanics and Microengineering (JMM), 25075017 doi:10.1088/0960-1317/25/7/075017.

[48] S. Khadraoui, M. Rakotondrabe and P. Lutz, ' Optimal design of piezoelectric cantilevered actuators with guaranteed performances by using interval techniques', IEEE/ASME - Transactions on Mechatronics (Tmech), Volume 19, Issue 5, Page 1660-1668, October 2014.

[49] M. Rakotondrabe and S. Khadraoui, 'Design of piezoelectric actuators with guaranteed performances using the performances inclusion theorem and interval tools', a chapter in 'Smart materials-based actuators at the micro/nano-scale: characterization, control and applications' edited by Micky Rakotondrabe, Springer - Verlag, New York, ISBN 978-1-4614-6683-3, 2013.

[50] M. Rakotondrabe, 'Performances inclusion for stable interval systems', ACC, (American Control Conference), pp.4367-4372, San Francisco CA USA, June-July 2011. 